Apidologie, 1978, 9 (4), 305-320.

\title{
DAS WÄRMEN DER BRUT BEI DER HONIGBIENE (APIS MELLIFERA L.)*
}

\author{
Le chauffage du couvain \\ par les abeilles (Apis mellifera L.)
}

\author{
N. KOENIGER \\ Fachbereich Biologie d.J.W. Goethe-Universität, \\ Institut für Bienenkunde (Polytechn. Ges.), Im Rothkopf 5, \\ D-6370 Oberursel/Ts.
}

\section{SUMMARY}

\section{THE WARMING OF HONEYBEE}

(APIS MELLIFERA L.) BROOD

In the experiments two brood samples were offered on a honey comb to $600-1200$ young worker bees. With thermocouples the temperature was registered. A comparison of different sorts of brood led to the following results :

1. Capped brood was warmed while the open brood was less attractive in all experiments.

2. Capped drone brood was five times more attractive than capped worker brood.

3. Two capped queen cells released warming behaviour if compared with 350 worker brood cells.

After removal of the pupa an empty queen cell was not warmed. The replacement of the pupa by a stone of similar weight did not inhibit warming behaviour. This mechanical stimulus alone however was not sufficient to elicit warming : queencells which were stored at $34^{\circ} \mathrm{C}$ for more than five hours were not active, when tested afterwards with an adequate stone. By adding extracts of pupae to these inactive cells warming behaviour could be released again. For recognition of the queen pupa two different complementary signals are necessary which were perceived as mechanical and chemical stimuli by the worker bees.

(*) Mit Ünterstützung der DFG. 


\section{ZUSAMMENFASSUNG}

Die Analyse des Wärmungsverhaltens wurde mit Hilfe von Simultanwahlversuchen ausserhalb des Nestes durchgeführt. Dabei bestand die Versuchsgruppe aus 600-1200 Arbeiterinnen. Über Thermoelemente wurden die Temperaturen der gebotenen Brutproben während des Versuchs registriert.

Ein Brutvergleich führte zu folgenden Ergebnissen :

1. Verdeckelte Brut wird verglichen mit offener Brut (Eier und Maden) bevorzugt gewärmt.

2. Verdeckelte Drohnenbrut zeigt eine ca. fünffache Reizwirksamkeit im Test gegen verdeckelte Arbeiterinnenbrut.

3. Zwei verdeckelte Weiselzellen werden gegen 350 verdeckelte Arbeiterinnenzellen bevorzugt gewärmt.

Nach dem Entfernen der Puppen erweisen sich leere Weiselzellen als unwirksam. Werden dagegen kurz vor Versuchsbeginn die Puppen durch einen gleich schweren Stein ersetzt, so kann mit Hilfe solcher "Steinzellen" Wärmungsverhalten ausgelöst werden. Dieser mechanische Reiz ist jedoch nicht allein wirksam. Zellen, die vor dem Versuch leer (ohne Puppen) bei $34^{\circ} \mathrm{C}$ für fünf Stunden aufbewahrt werden, lösen, mit einem adäquaten Stein getestet, niemals Wärmungsverhalten aus. Erst wenn diese inaktiven Zellen zusätzlich mit Puppenextrakt versehen werden, wird gewärmt. Für die Wärmung von Königinnenzellen sind demnach zwei komplementäre Signale der Puppe erforderlich, die als mechanische und chemische Reize von den Arbeiterinnen wahrgenommen werden.

\section{EINLEITUNG}

Die Thermoregulation im Volk der Honigbiene ist schon seit langer Zeit bekannt (ARISTOTELES 384-322 v. Chr.). Über die Temperaturverteilung innerhalb des Bienenvolkes liegt eine grosse Anzahl von Untersuchungen vor (Gates 1914, Hess 1926, Himmer 1927 a, Free + Spencer-Booth 1958, Büdel + Herold 1960, Esch 1968 u.a.), die z.T. sehr genaue Beschreibungen über Temperaturverhältnisse bei verschiedenen Umweltbedingungen enthalten. Die verhaltensphysiologischen Grundlagen dieses Phänomens sind jedoch bis heute weitgehend unbekannt.

So gibt es kernerlei Angaben über das "Wärmungsverhalten " der Bienen im Volk. Auch die genauen Untersuchungen von Rösch (1925) und von Lindauer (1952), bei denen u.a. einzelne Bienen über lange Zeiträume intensiv beobachtet wurden, geben hier keinen Aufschluss. Dennoch fällt auf, dass nach Lindauer (1952) die beobachtete Stockbiene über die Hälfte ihrer Zeit mit "Nichtstun " verbringt. Es erscheint daher nicht unmöglich, dass die Biene eine gewisse Zeitspanne ihres Lebens mit Wärmeerzeugung verbringt, und dass dieses Verhalten so wenig auffällig ist, dass es von einem Beobachter ohne Zuhilfenahme empfindlicher Temperaturfühler nicht bemerkt werden kann.

Aber auch in anderer Hinsicht sind die verhaltensphysiologischen Grundlagen des Wärmens bei der Honigbiene noch wenig geklärt. So werden zwischen den Temperaturverhältnissen im Bienenvolk während des Winters und des Sommers grosse Unterschiede beschrieben: Während der brutlosen Zeit (Winter) schwanken die Temperaturen in einem Bereich von $24^{\circ}-34^{\circ} \mathrm{C}$. Im Sommer dagegen, beim Auftreten der ersten verdeckelten Brutzellen (Puppen), zeigen sich wesentlich konstantere Verhältnisse. Die Brutnesttemperatur wird auf $34,5 \pm 0,3{ }^{\circ} \mathrm{C}$ eingestellt (HiMmER 
1927 a). Eine Erklärung für diese Unterschiede kann z.Zt. nur ansatzweise gegeben werden. Ritrer (1975) konnte zeigen, dass bei seinen Versuchen während des Sommers die Thermoregulation in einem Bienenvolk ohne Brut und in einem vergleichbaren Volk, das verdeckelte Brutzellen enthält, ähnliche Unterschiede in der Temperaturverteilung aufweist, wie sie für ein Wintervolk und ein Sommervolk beschrieben sind. So liegt est nahe zu vermuten, dass die genaue Thermoregulation im Sommer direkt durch die Anwesenheit von Puppen ausgelöst wird. Eine weitere Bestätigung für diese Hypothese - die Temperaturverteilung im Sommerbienenvolk wird weitgehend durch das aktive Wärmen der Brut von den Arbeitsbienen bestimmt kann aus genauen Temperaturmessungen bei Kühlungsversuchen abgeleitet werden. So lag die direkt an den Puppen gemessene Temperatur bei diesen Versuchen statistisch signifikant über den Temperaturen, die zwischen den Waben innerhalb des Bienensitzes registriert wurden (RITTER und KoENIGER 1977).

Über das Erkennen der Puppen durch die Imagines bei Apis liegen bisher keine Arbeiten vor. Während sich bei den Ameisen der Transport der Puppen als eine gute natürliche Basis für entsprechende Analysen erwiesen hat, fehlt bei Bienen und Wespen dieses Verhalten. So soll versucht werden, das aktive Wärmungsverhalten der Imagines als Indikator für die " Erkennung der Brut " heranzuziehen.

Es wird zunächst darauf ankommen, dieses Wärmungsverhalten unter kontrollierten Bedingungen, d.h. ausserhalb der Nester, auszulösen. Mit Hilfe eines geeigneten Biotests soll die unterschiedliche Attraktivität von Eiern, Maden und Puppen bzw. von Drohnen-, Arbeiterinnen- und Königinnenpuppen verglichen werden. Weiter werden die Faktoren analysiert, die bei den Arbeiterinnen das Wärmungsverhalten auslösen.

\section{MATERIAL UND METHODEN}

\section{Haltung der Bienen}

Für die Versuche standen in den Jahren 1970-1977 je zwölf Völker der Rasse Apis mellifica carnica zur Verfügung. Im allgemeinen wurden Kombinationen der Linie $07 \times 1075$ sowie $1012 \times 1075$ zur Erstellung der Königinnen benutzt. Den Königinnen wurde dann der Hochzeitsflug in Oberursel gestattet, so dass die in den Versuchen eingesetzten Bienen Bastarde zwischen reinen Apis mellifica carnica und der Landrasse (Drohnen) sind. Diese Bienen zeichnen sich im allgemeinen durch eine besondere Wabenstetigkeit aus, die für die Entwicklung der Biotests von grosser Bedeutung war. Die zwölf Bienenvölker waren im Freiland aufgestellt und befanden sich 10-20 m von den Räumen entfernt, in denen Versuche durchgeführt wurden.

\section{Biotest}

Der Biotest war als Simultanwahlversuch konzipiert. In eine mit Honig gefüllte Bienenwabe wurden die beiden $\mathrm{zu}$ untersuchenden Brutproben eingeschnitten und die so präparierte Wabe in einen mit Drahtgitter bespannten Versuchskasten eingebracht. Dort wurden Thermoelemente jeweils an die Brutproben sowie ein drittes Thermoelement auf die Honigwabe in der Mitte zwischen beiden Brutproben befestigt. Für den Versuch wurden nun aus einem der Bienenvölker zwei bis vier mit Bienen besetzte Brutwaben entnommen, für ca. 15 Min. im Freien belassen, und dann die Bienen in einen leeren Kasten 
abgekehrt. Nach drei bis fünf Stunden Aufbewahrung unter den gleichen Temperaturbedingungen wie im Test wurden die Bienen in den Versuchskasten gegeben. Dort liefen die Arbeiterinnen sofort auf die Honigwabe und begannen Honig aufzunehmen (Abb. 1 a). In diesem Stadium waren die Bienen nahezu gleichmässig auf die Wabe verteilt. Etwa 15 Min. später setzte bei den Tieren eine Phase der Unruhe ein und ca. $40-80 \%$ der Tiere verliessen den Versuchsaufbau und flogen in ihr Volk zurück. Dabei handelte es sich um die älteren, bereits in der Flugphase befindlichen Tiere. Die restlichen verbliebenen Bienen sammelten sich dann auf den Brutproben, wobei sehr bald ein Ungleichgewicht entstand (Abb. 1 b), d.h. die Anzahl der Bienen auf der attraktiveren Brutprobe nahm zu. Nach ca. 2-5 Std. wurde in der Regel nur noch eine Probe belagert (Abb. 1 c). Über die auf der Wabe angebrachten Thermoelemente wurden die Temperaturen registriert. Diese Kurven erlaubten eine Analyse der Temperaturverteilungen während des Testes (Abb. 2). Abweichungen vom beschriebenen Verhalten konnten nicht selten beobachtet werden. So verliessen bei ca. $15 \%$ der Versuche sämtliche Bienen die Versuchsanordnung und bei anderen Biotests ( $5 \%)$ blieben so viele Bienen zurück, dass beide Brutproben gewärmt wurden. In diesen Fällen bildete sich eine durchgehende Traube, die sich von der einen Probe über den $Z$ wischenraum hinweg bis auf die andere Brutprobe erstreckte.

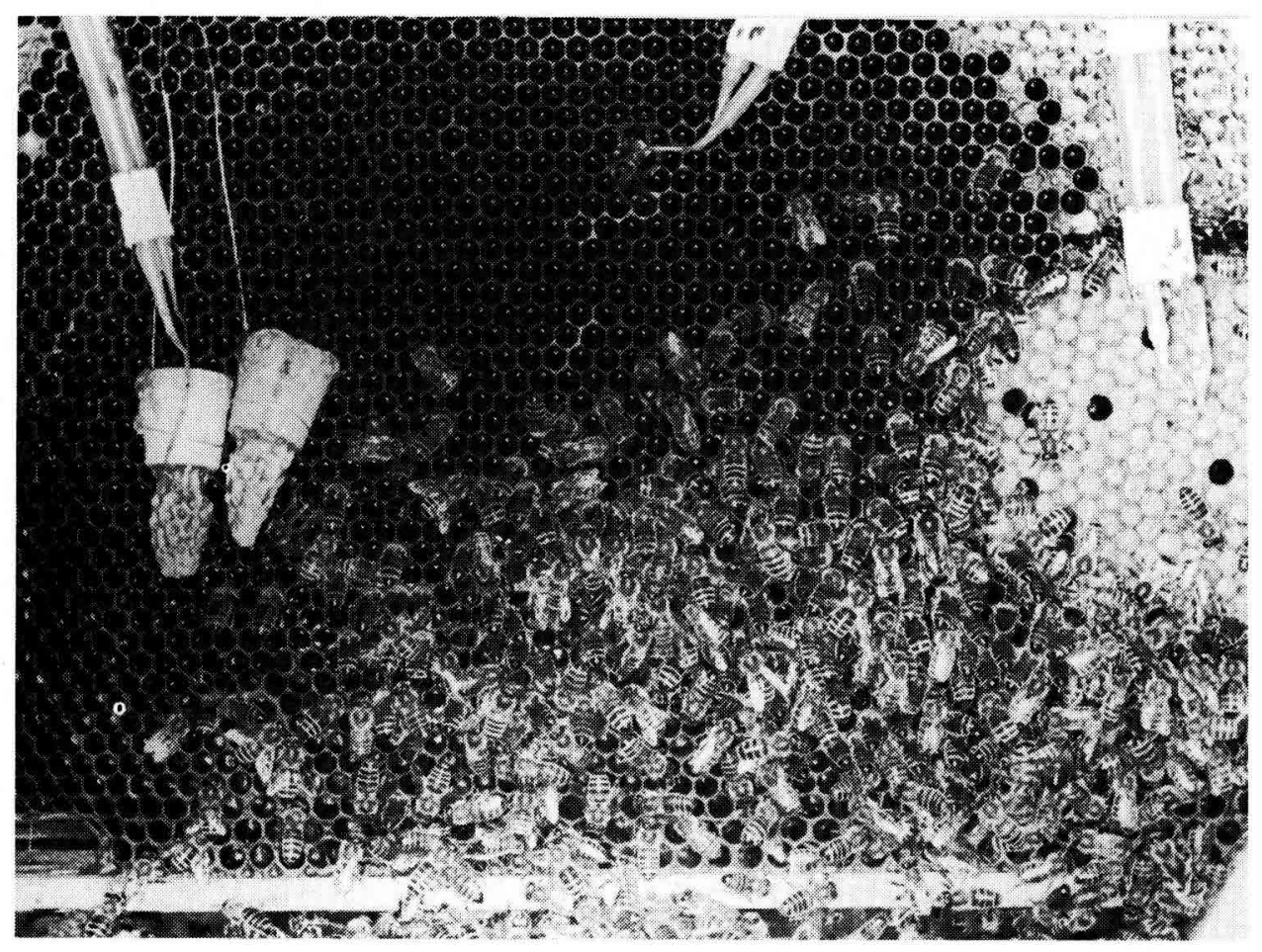

ABB. 1. - Biotest. Vergleich zwischen zwei leeren Königinnenzellen und 350 verdeckelten Arbeiterinnenzellen.

FIG. 1. - Biotest. Comparaison entre 2 cellules royales vides et 350 cellules operculées d'ouvrières.

Aвв. 1 a). $-Z$ wei Minuten nach Versuchsbeginn:

Die Bienen laufen auf die Wabe und nehmen Honig auf.

Fig. 1 a). - Deux minutes après le début de l'expérience: Les abeilles courent sur le rayon et prélèvent du miel. 


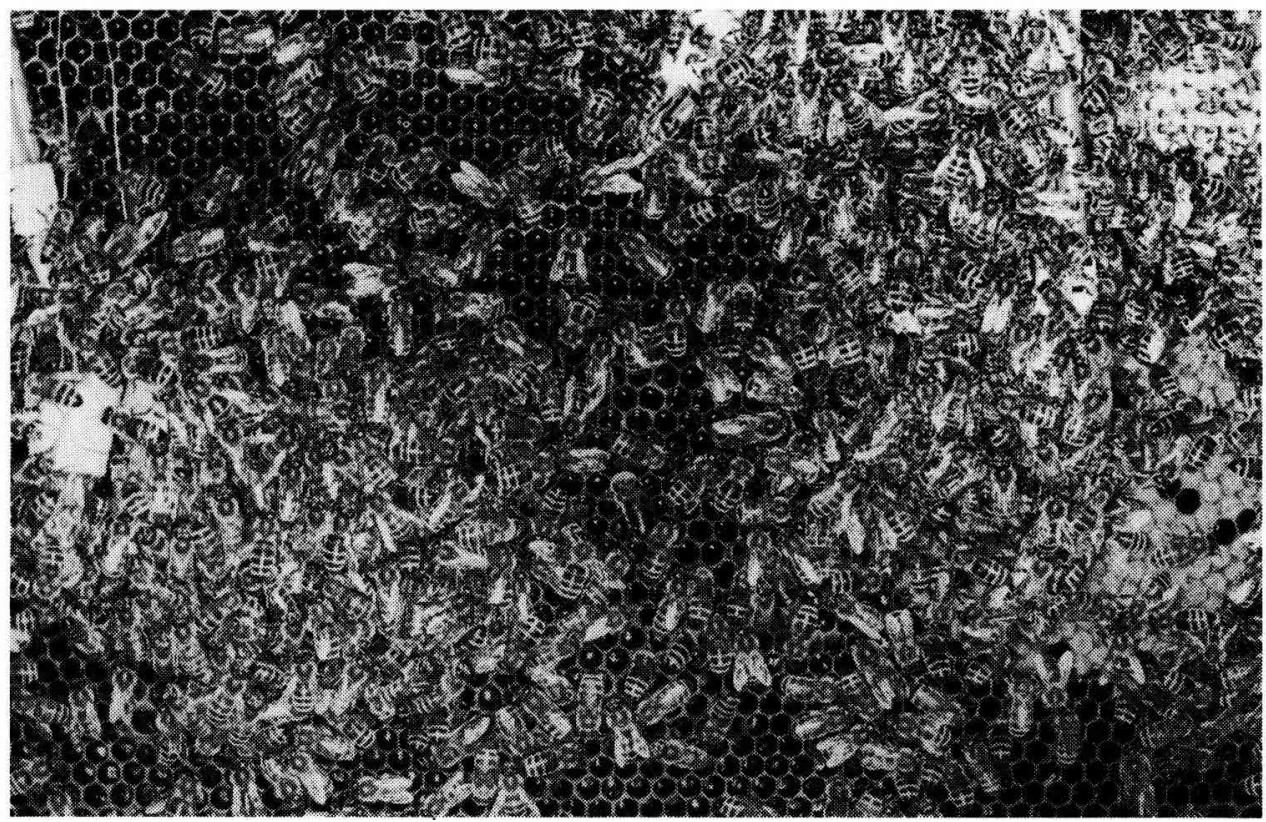

AвB. 1 b). - 40 Minuten nach Versuchsbeginn:

Ein Teil der Bienen hat die Wabe verlassen. Auf beiden Proben konzentrieren sich dic restlichen Tiere.

FIG. 1 b). - 40 minutes après le début de l'expérience:

une partie des abeilles a quitté le rayon.

Celles gui restent sont concentrées sur les deux échantillons.



Aвв. 1 c). - 2 Stunden nach Versuchsbeginn:

Nur noch eine Probe wird belagert und gewärmt.

FIG. $1 \mathrm{c})$. - Deux heures après le début de l'expérience:

Un échantillon est encore assiégé et chauffé. 
Die Versuche wurden in den Abendstunden angesetzt (zwischen $17.30 \mathrm{~h}$ und $19.00 \mathrm{~h}$ ) und liefen bis zum nächsten Morgen. Die Temperatur in den Versuchsräumen wurde zwischen $14^{\circ} \mathrm{C}$ und $18^{\circ} \mathrm{C}$ konstant gehalten. Weiter waren die Räume dunkel. Lediglich durch den Laufkanal, der den Versuchskasten mit dem Freiland verband, gelangte in den Abend- und Morgenstunden Licht in die Versuchsanordnung.

\section{Messtechnik}

Die Registrierung der Temperatur während der Biotests erfolgte durch einen 12-PunkteKompensationsschreiber, Typ Polycomb 2 (Firma Hartmann und Braun). Die Temperatur wurde über selbstgebaute Thermoelemente gemessen (Abb. 3).

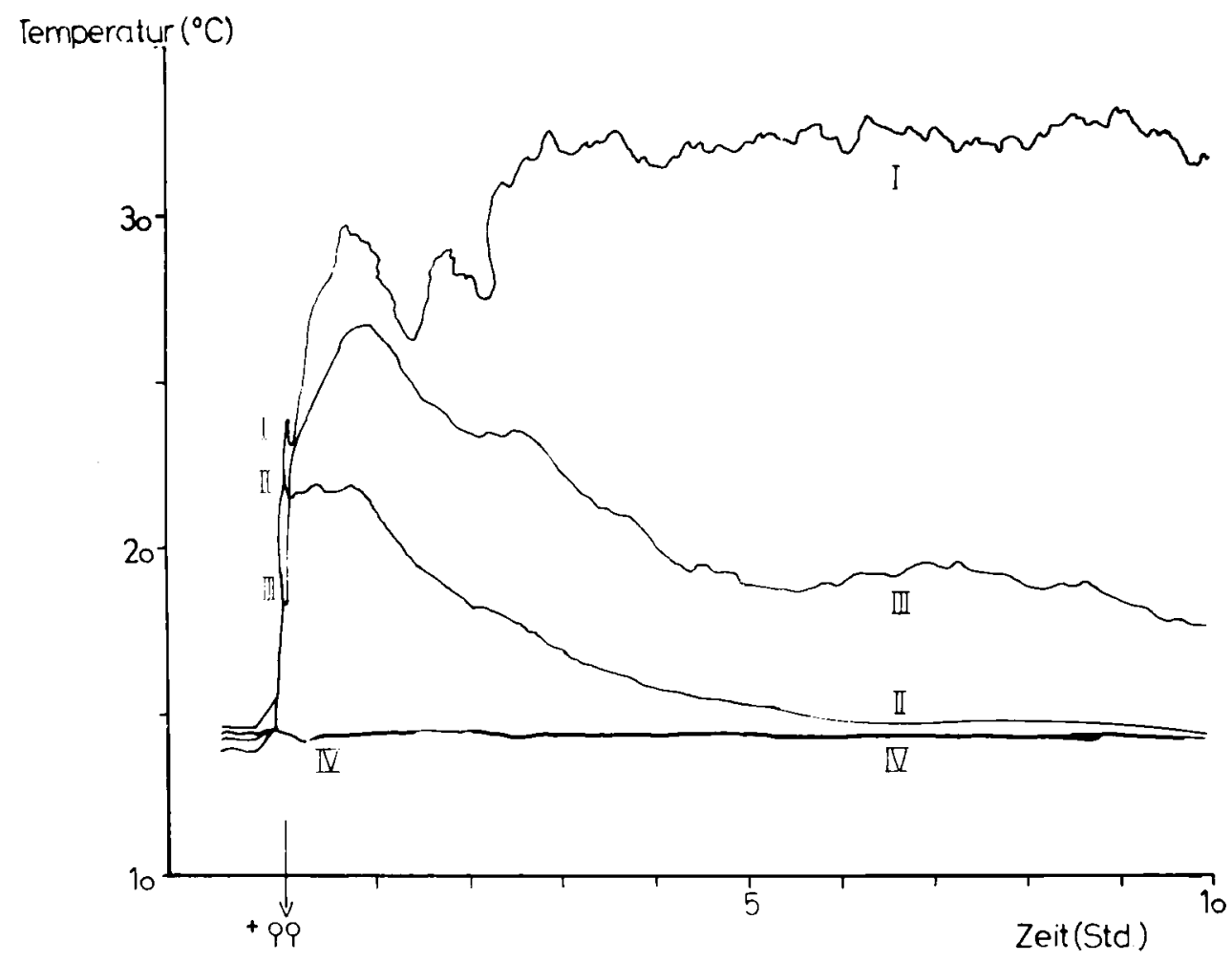

Aвв. 2. - Temperaturverteilung während des Biotests.

I Temperatur der Arbeiterinnenbrutzellen.

II Temperatur der leeren Königinnenzellen.

III Temperatur der Honigwabe zwischen beiden Proben.

IV Umgebungstemperatur.

FIG. 2. - Répartition des températures durant le biotest.

I Température des cellules d'ouvrières.

II Température des cellules royales vides.

III Température du rayon à miel entre les deux échantillons.

IV Température ambiante. 


\section{ERGEBNISSE}

\section{Attraktivitätsvergleich}

Offene gegen verdeckelte Brut

Im Biotest wurden verschiedene Proben offener Brut (Eier und Maden) gegen verdeckelte Brut geboten. Dabei wurde die Anzahl der verdeckelten Brutzellen konstant gehalten; sie betrug bei allen Versuchen 350 Zellen \pm 10 . Die Anzahl der Eier und Maden dagegen schwankte zwischen 350 und 700 . Bei allen 13 Versuchen besetzten die Bienen nur die verdeckelte Brut. Die offene Brut wurde in keinem Fall gewärmt.

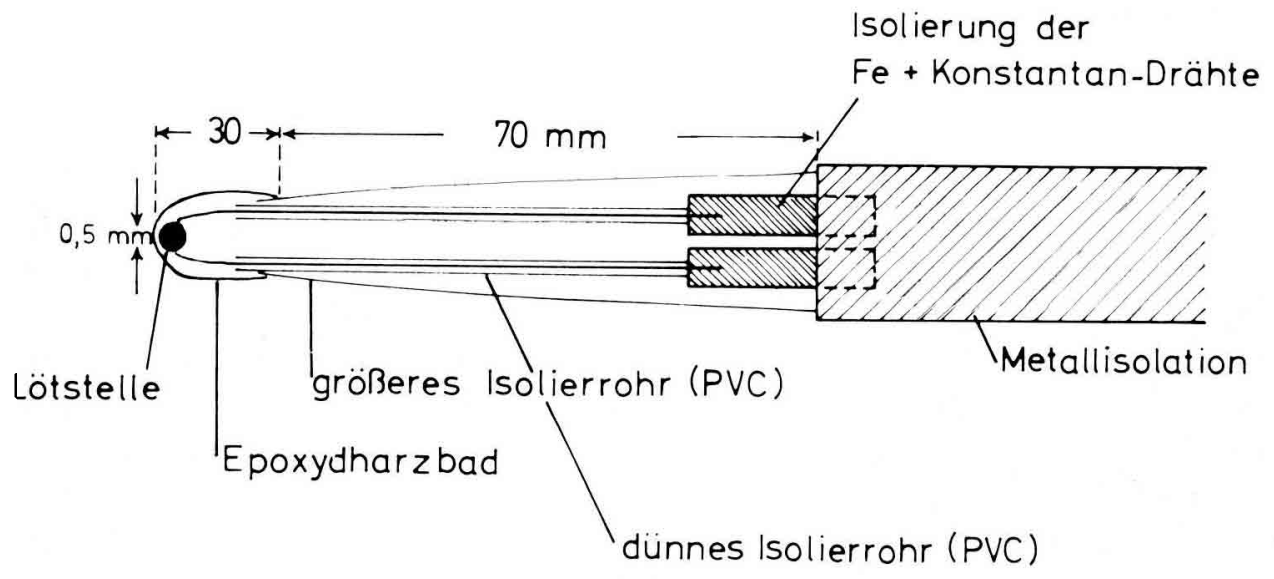

Aвв. 3. - Querschnitt der verwendeten Thermoelemente.

Fig. 3. - Vue en coupe du thermocouple.

De gauche à droite : Point de soudure: bain de résine d"époxyde: grosse gaine d'isolation (PVC): fine gaine d'isolation (PVC): Isolation des fils de fer et de constantan: isolation métallique.

\section{Drohnenpuppen gegen Arbeiterinnenpuppen}

In fünf Versuchen wurden 350 verdeckelte Arbeiterinnenzellen mit der gleichen Anzahl verdeckelter Drohnenzellen verglichen. In allen Fällen wurde die verdeckelte Drohnenbrut gewärmt. Daraufhin wurde bei gleichbleibender Arbeiterinnenprobe die Anzahl der gebotenen Drohnenzellen verringert. In vier Fällen wurden 100 verdeckeite Drohnenzellen gegenüber 350 verdeckelten Arbeiterinnenzellen bevorzugt. Bei sieben weiteren Versuchen wurden dann 75 Drohnenzellen eingesetzt mit dem Ergebnis, dass dreimal die Drohnen- und viermal die Arbeiterinnenbrut gewärmt wurde.

\section{Königinnenpuppen gegen Arbeiterinnenpuppen}

Bei 14 Versuchen wurden 350 Arbeiterinnenzellen jeweils gegen 2 normale, intakte Königinnenzellen geboten. In allen Fällen wurden die Königinnenzellen gewärmt. 


\section{Leere Zellen}

Leere Königinnenzellen, aus denen ca. lo Min. vor Versuchsbeginn die Puppen entnommen wurden, wurden mit 350 verdeckelten Arbeiterinnenzellen verglichen (Abb. 1). In allen sieben Versuchen wurden die Arbeiterinnenzellen gewärmt, und es konnten lediglich einzelne Bienen beobachtet werden, die während des Versuchs zu den Königinnenzellen hinliefen, dort aber nicht länger verweilten. Meist schon innerhalb von ca. $15 \mathrm{Min}$. kehrten sie wieder zur Traube der wärmenden Bienen auf der Arbeiterinnenbrut zurück (Abb. 1 c). Nach diesem Ergebnis scheinen die Bienen also in der Lage zu sein, zu erkennen, ob eine Zelle leer ist.

\section{"Steinzellen 》}

In den nächsten 9 Versuchen wurde jeweils nach Entfernen der Puppe aus der Königinnenzelle ein Stein in die Zelle gelegt, dessen Gewicht (175-220 mg) dem der Königinnenpuppen entsprach. In allen Versuchen wurden die Königinnenzellen gewärmt und nicht die 350 Arbeiterinnenzellen. Daraufhin wurden in drei Versuchen leere Königinnenzellen mit normalen Königinnenzellen verglichen. Dabei waren sowohl an den leeren wie auch an den mit Puppen gefüllten Königinnenzellen Tonabnehmer angebracht, die über einen Verstärker die auftretenden Schwingungen auf einem Tonband aufzeichneten. Die Analyse dieser Aufnahmen ergab eine Fülle von sehr verschiedenen Schwingungen. Einen systematischen Unterschied zwischen leeren und gefüllten Königinnenzellen konnten wir jedoch nicht bemerken. So wurde versucht, durch andere Anordnungen näheren Aufschluss über den Unterscheidungsmechanismus zwischen leeren und vollen Zellen $\mathrm{zu}$ erhalten.

\section{Styroporzellen}

Die Vergleichsprobe von 350 verdeckelten Arbeiterinnenzellen wurde mit frischen Königinnenzellen getestet, die mit kleinen Styroporsäulen gefüllt wurden. Das Gewicht dieser Styroporzellen war in allen Fällen unter $10 \mathrm{mg}$. In drei Versuchen, in denen die Styroporkörper mit ihrer Längsachse parallel zur Längsachse der Königinnenzellen plaziert waren (Abb. 4 rechts), wurde die Arbeiterinnenbrut gewärmt.

In fünf Fällen wurden die Styroporkörper in ihrer Längsachse zusammengedrückt und in die Königinnenzellen hineingezwängt (Abb. 4, links), so dass nun die ursprüngliche Längsachse des Styroporkörpers senkrecht auf der Längsache der Königinnenzelle stand. Von diesen 5 Versuchen wurden viermal die Königinnenzellen und einmal die Arbeiterinnenzellen gewärmt. Diese Ergebnisse lassen die Vermutung zu, dass der Druck des Zellinhalts auf die Zellwand von den Bienen für die nachgewiesene Unterscheidung zwischen leeren und vollen Königinnenzellen benutzt wird. 


\section{Pendelpuppen}

Zur Überprüfung dieser Hypothese wurde nun bei Königinnenzellen die Puppe aus ihrer natürlichen Position entfernt und vor Versuchsbeginn mit der Abdomenspitze oben in der Königinnenzelle festgeklebt (Abb. 5). Auf diese Weise hingen die Puppen, vergleichbar mit einem Pendel, frei in den Zellen und konnten keinerlei Druck auf die Zellwand ausüben. In fünf Versuchen wurden Zellen mit "Pendelpuppen" gegen

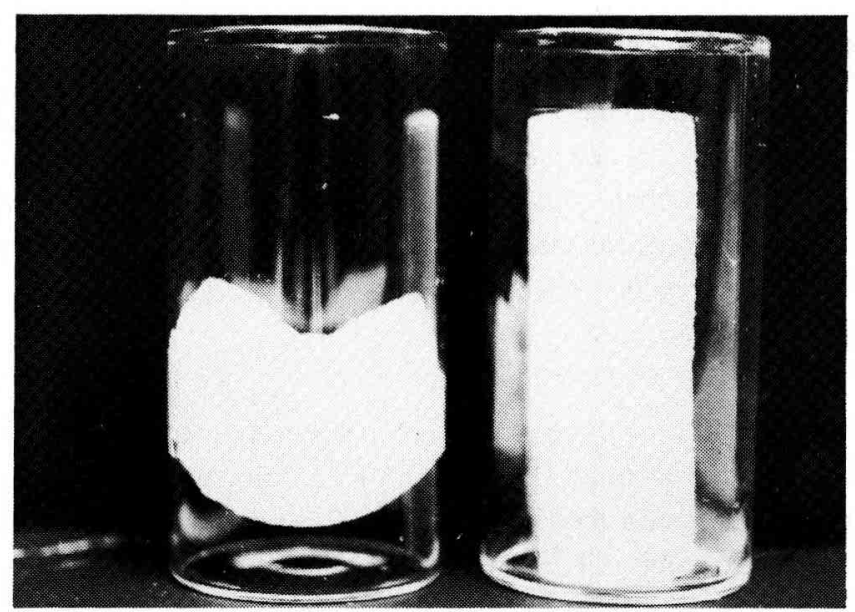

Авв. 4. - Darstellung zur Demonstration der Position der Styroporquader.

Die im linken Glas dargestellte Position erwies sich als reizwirksam.

FIG. 4. - Influence de la position du bloc de polystyrène expansé.

La position représentée dans le verre de gauche se trouve être stimulante.

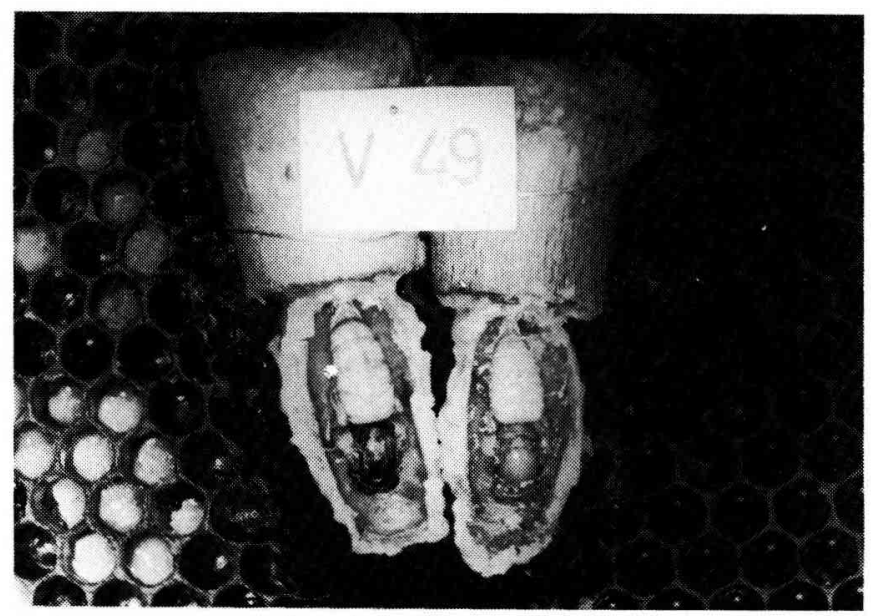

ÀBB. 5. - Pendelipuppe.

Die Königinnenpuppen wurden mit dem Abdomen oben am Zellgrund festgeklebt.

Fig. 5. - Pupe-pendule.

Les pupes de reines ont été collées par l'abdomen sur le fond supétieur de la cellule. 
jeweils 350 verdeckelte Arbeiterinnenzellen geboten. In allen Versuchen wurden die Arbeiterinnenzellen gewärmt.

\section{Analyse des chemischen Auslösers}

In den folgenden Versuchen wurden nun zwei Proben miteinander verglichen, die jeweils aus zwei Königinnenzellen bestanden.

\section{Frische Zellen gegen alte Zellen}

Aus zwei Königinnenzellen wurden die Puppen entfernt und die Zellen 424 Stunden in einem Brutschrank bei $34^{\circ} \mathrm{C}$ aufbewahrt. In 7 Versuchen wurden diese Königinnenzellen, nachdem sie mit einem Stein gefüllt waren, mit zwei Königinnenzellen verglichen, bei denen 10 min vor Versuchsbeginn die Puppen durch Steine ersetzt wurden. In allen sieben Versuchen wurden die frischen Zellen gewärmt.

\section{Reaktivierung der Königinnenzellen}

In einer Versuchsserie wurden Königinnenzellen getestet, deren Aktivität durch eine fünfstündige Aufbewahrung (leer) bei $34^{\circ} \mathrm{C}$ aufgehoben worden war. Diese Königinnenzellen wurden nun jeweils drei, zehn, vierundzwanzig und achtundvierzig Stunden vor Versuchsbeginn mit lebenden Puppen gefüllt und im Versuch mit zwei normalen intakten Königinnenzellen verglichen. Bei 6 Versuchen, bei denen die Reaktivierungszeit weniger als 24 Stunden betrug, wurden jeweils nur die intakten Vergleichszellen gewärmt. Bei 3 Versuchen mit 48 stündiger Reaktivierungszeit wurden die reaktivierten Zellen gewärmt.

\section{Extraktionsversuche}

Arbeiterinnen- und Königinnenpuppen wurden jeweils 10-20 min mit Diäthyläther im Soxlethapparat extrahiert. Für den Test der Extrakte wurde folgende Anordnung gewählt : Extrakte aus 15-30 Königinnen- bzw. 300-700 Arbeiterinnenpuppen wurden bei Zimmertemperatur auf Watte eingedampft. Vor dem Test wurde dann die mit Extrakt kontaminierte Watte auf zwei Königinnenzellen verteilt und diese Zellen für fünf Stunden bei $34^{\circ} \mathrm{C}$ aufbewahrt. Im Versuch wurden diese Zellen mit zwei gleich behandelten Königinnenzellen ohne Extrakt verglichen. In elf Fällen wurden die Zellen, die den Extrakt enthielten, gewärmt. In einem Fall jedoch die Kontrollzellen.

\section{DISKUSSION}

\section{Biotests}

Das Wärmen der Puppen ist bei der Honigbiene unter natürlichen Bedingungen an das Volk gebunden. Im Bienenvolk jedoch ergeben sich für experimentelle Arbeiten Schwierigkeiten. Das grosse Regulationsvermögen und die Variabilität der Reaktion 
auf Störungen stehen der erforderlichen Reproduzierbarkeit der Versuche entgegen. Weiter erfordert die Erfassung und Registrierung der Bienenbewegungen und der Temperaturen einen grossen apparativen Aufwand. Für die experimentelle Arbeit war es daher günstiger, ausserhalb des Volkes unter besser zu kontrollierenden Bedingungen zu arbeiten. Dabei mussten jedoch folgende Punkte beachtet werden : $\mathrm{Zu}$ nächst durfte eine Gruppengrösse von ca. 600 Arbeiterinnen nicht unterschritten werden, da kleinere Gruppen in der Regel überhaupt nicht auf Brut reagieren, sondern in einer Ecke des Kastens eine Traube bilden. Die Anzahl der verbleibenden Bienen muss zwischen 600 und 1200 liegen, da in diesem Fall nur eine Brutprobe gewärmt wird. Eine grössere Menge von Bienen (über 1500 ) bedeckt in der Regel beide Brutproben und den dazwischen liegenden Teil der Testwabe. In diesen Fällen sind nur kleine Temperaturdifferenzen zwischen den Brutproben zu beobachten.

Ferner war es notwendig, für den Test ausschliesslich Tiere zu benutzen, die eine ausreichend grosse " Motivation » zum Brutwärmen hatten. Bienen, die nicht im Sinne des Testes reagierten, liefen unruhig hin und her, und es gelang ihnen oft, ihre Unruhe auf die zunächst ruhigen Tiere, die sich auf der Brut gesammelt hatten, zu übertragen. Nach einer mehrstündigen Unruhe sammelten sich die Bienen dann in einer Traube und beachteten die Brut nicht. Deshalb wurde die Auswahl der Versuchstiere in zweifacher Weise durchgeführt. Zunächst wurden mit Bienen besetzte Brutwaben aus dem Volk genommen und für $15 \mathrm{~min}$ im Freien belassen, so dass während dieser Zeit ein Teil der Bienen abfliegen konnte. Zum zweiten wurde im Versuchskasten ein Ausflug. angebracht, durch den Bienen ins Freiland und zurück in das Volk gelangten, aus dem sie gekommen waren. Auf diese Weise verblieben nur die brutwärmenden Tiere und alle anders motivierten älteren Bienen entfernten sich, ohne den Versuch zu stören.

Ein Nachteil dieser Technik bestand darin, dass nicht genau vorauszusehen war, wieviele der eingebrachten Bienen " freiwillig " im Test bleiben würden. Hier zeigten sich sehr grosse Unterschiede zwischen den verschiedenen Völkern, die getestet wurden. Durch die Verwendung von genetisch einheitlichen Linien gelang es jedoch, diese Schwierigkeiten zu mindern. Diese Bienen, die sich durch guten "Wabensitz " und Sanftmut auszeichneten, ermöglichten den reproduzierbaren Test. Zwischen 30 und $50 \%$ der Tiere verblieben in der Regel, sofern die gebotenen Brutproben eine ausreichende Attraktivität besassen. Unter den herrschenden Bedingungen mussten immer mindestens ca. 350 verdeckelte Arbeiterinnenzellen oder eine entsprechende Menge anderer Puppen im Versuch vorhanden sein, um eine genügende Anzahl von Bienen zum Bleiben zu veranlassen.

Zusammenfassend kann gesagt werden, dass aufgrund des hier geschilderten Biotests eindeutige Aussagen möglich sind. Die Versuchsbienen entschieden sich unter den o.a. Bedingungen alternativ für eine Brutprobe und wärmten diese. Die Temperaturen schwankten je nach Raumtemperatur auf den gewärmten Proben zwischen $31^{\circ}$ und $35^{\circ} \mathrm{C}$. 


\section{Attraktivitätsvergleich}

In den Versuchen wurde offene Brut von den Bienen weitaus schlechter bewertet als verdeckelte Brutzellen. Wurde im Test ausschliesslich offene Brut geboten, so verliessen die Arbeiterinnen den Testkasten oder bildeten sehr häufig Trauben am Deckel des Kastens. Der Befund, dass verdeckelte Zellen vorzugsweise gewärmt werden, stimmt gut mit den Beobachtungen bei der imkerlichen Praxis überein. Wird eine Wabe aus dem Volk genommen und auf den Wabenbock gestellt, so verlassen die Bienen in der Regel die Wabe, wenn keine verdeckelte Brut vorhanden ist. Wabenbezirke mit verdeckelter Brut dagegen werden dicht belagert. Die "Zweckmässigkeit " dieses Verhaltens lässt sich anhand von Unterkühlungsversuchen gut demonstrieren. Eier und Larven zeigen nach unseren Ergebnissen selbst bei fünfstündiger Aufbewahrung bei $10^{\circ} \mathrm{C}$ nur geringe Schäden : ca. $10 \%$ wurden nach Zugabe der gekühlten Wabe in ein Volk von den Bienen entfernt. Bei verdeckelter Brut dagegen zeigte sich bei der gleichen Behandlung, dass die Sterberate etwas niedriger lag (7\%). Bei den geschlüpften Bienen allerdings waren bei $70 \%$ der Tiere Schäden festzustellen. Meist traten Malformationen an Flügeln und Extremitäten auf. Schätzungsweise $60-80 \%$ der geschlüpften Bienen waren aufgrund der aufgetretenen Schäden an den Flügeln nicht flugfähig. Himmer (1927 a) und Weiss (1962) kamen bei Kühlung von Puppen zu entsprechenden Ergebnissen.

Verdeckelte Drohnenzellen scheinen etwa fünfmal attraktiver als verdeckelte Arbeiterinnenzellen zu sein. $\mathrm{Zu}$ diesem Ergebnis muss bemerkt werden, dass die entsprechenden Versuche nur während der Zeit durchgeführt wurden, in der natürlicherweise Drohnenbrut vorhanden ist (Mai bis Anfang Juli). Weiter muss bei der Interpretation berücksichtigt werden, dass Bienen, bevor sie in den Test kamen, seit mindestens 5 Stunden von ihrer Königin getrennt waren. Es ist seit langem bekannt, dass weisellose Bienenvölker Drohnenbrut aufziehen und die geschlüpften Drohnen im Volk pflegen, auch zu Zeiten, in denen weiselrichtige Völker natürlicherweise keine Drohnen mehr erzeugen und auch keine Drohnen mehr in ihren Völkern dulden.

Verdeckelte Königinnenzellen zeigen im Test die grösste Überlegenheit. Zwei Königinnenzellen wurden in allen Fällen 350 Arbeiterinnenzellen vorgezogen. Wie zwei Kontrollversuche vermuten lassen, in denen die Bienen mit ihrer Königin in den Test gegeben wurden, lässt sich dieses Phänomen wohl nicht ausschliesslich mit der Weisellosigkeit der Versuchsbienen erklären, denn auch bei Anwesenheit der Königin wurden die Weiselzellen gewärmt.

\section{Wärmungsverhalten an Königinnenzellen}

\section{Mechanische Auslöser}

Leere Königinnenzellen werden, wie die Ergebnisse zeigen, von den Bienen im Vergleich zu vollen Königinnenzellen nicht gewärmt. Damit erscheint es sicher, dass die Bienen über einen Perzeptionsmechanismus verfügen, der sie in die Lage versetzt, den Zellinhalt wahrzunehmen. Zunächst konnte gezeigt werden, dass ein Stein, der 
gleich schwer ist wie die Königinnenpuppe, in einer frischen Königinnenzelle geboten, Wärmungsverhalten auslöst. In zwei Versuchen wurden nun die im Test auftretenden Schwingungen und Vibrationen von Königinnenzellen abgeleitet, die leer, mit einem Stein oder einer Puppe gefüllt waren. Eine Analyse mit Hilfe eines Oszillographen, sowie das Abhören der entsprechenden Aufnahmen ergaben keinerlei Anhaltspunkte für systematische Unterschiede zwischen leeren und gefüllten Zellen.

Da uns zur Zeit eine geeignete Messmethode fehlt, mit deren Hilfe eine genaue Analyse der Schwingungsvorgänge der Zellwand möglich ist, wurde zunächst eine Eingrenzung auf anderem Wege versucht. So erhielten wir bei den Versuchen mit Styroporkörpern einen Hinweis, dass die Spannung der Zellwand, die durch das Gewicht der Puppe oder des Steines entsteht, bei der Erkennung des Zellinhalts entscheidend sein kann. Als Bestätigung für diese Überlegung können die Versuche mit Pendelpuppen angeführt werden. Sie zeigen, dass Zellen im Test keinerlei Wärmungsverhalten auslösen, wenn Puppen keinen Druck auf die Zellwand ausüben.

Bei ihren Untersuchungen über den Wabenbau beschreiben MARTIN und LiNDAUER (1966) eine Hypothese für die Perzeptionsvorgänge, die dem massgerechten Bau der Zellwand - insbesondere der Wanddicke - zugrunde liegen : Mit den Mandibeln wird von der Baubiene die Zellwand eingedellt und die Rückschwingung der Wand mit Hilfe der Antennen registriert. Auf diese Weise ist es den Bienen möglich, die Wanddicke der Zelle auf wenige hundertstel Millimeter genau zu regulieren (Arbeiterinnenzellen $\pm 1.57 \mu$; Drohnenzellen $\pm 2,35 \mu$ ). Ein derartiger Perzeptionsmechanismus würde sicher eine Unterscheidung zwischen leeren Königinnenzellen und Königinnenzellen, in denen der Inhalt (Puppe oder Stein) einen Druck auf die Zellwand ausübt, ermöglichen.

\section{Chemische Auslöser}

Der Nachweis von chemischen Auslösern gelang mit Diäthylätherextrakt. Eine weitere chemische Auftrennung dieser Extrakte, die zu der Identifizierung der aktiven Substanz(en) führen soll, ist z.Zt. in Arbeit.

\section{Das Wärmen der Puppenzellen im Bienenvolk}

Eine Trennung der wirksamen Reize in Bezug auf verschiedene Funktionen ist bisher noch nicht erkennbar. Eine systematische Gliederung des gesamten Verhaltensablaufes kann dieses verdeutlichen. Das Gesamtverhalten lässt sich in drei verschiedene Abschnitte unterteilen :

1. Auffinden der Puppenzelle.

2. Verbleiben bei der Puppenzelle.

3. Erzeugung und Übertragung von Wärme auf die Puppenzelle.

Die Puppenzelle wird im Volk von den Bienen auch ohne Kommunikation über Entfernung aufgefunden. Nach Lindauer (1952) «patroullieren " ständig eine grössere Anzahl Arbeiterinnen durch das Volk, so dass sie zwangsläufig dabei auf Puppenzellen treffen. 
Das Verbleiben bei der Puppenzelle war bei der hier angewandten Versuchsmethode in jedem Fall mit der Erzeugung und Übertragung von Wärme gekoppelt. Weiter konnte das Wärmungsverhalten nur durch Puppenzellen ausgelöst werden, bei denen die mechanischen und chemischen Voraussetzungen gleichzeitig vorhanden waren. Auch in dieser Hinsicht ist die Koppelung zweier Faktoren eine notwendige Bedingung. Die Ergebnisse erlauben die Vermutung, dass nur beide Auslöser gemeinsam wirksam sind.

Eine schematische Übersicht (Abb. 6) zeigt den funktionellen Zusammenhang der wichtigsten an der Thermoregulation beteiligten Verhaltenskomponenten. In den Völkern der Bienen sind auf engem Raum viele Tiere vereinigt, deren Stoffwechsel zwangsläufig eine beträchtliche Wärmemenge erzeugt. Eine Regulation wird in erster Linie bei kleineren Temperaturschwankungen durch die Verteilung der Arbeiterinnen im Nest erreicht. Durch eine Erhöhung der Dichte der Arbeiterinnen wird die abgegebene Wärmemenge, bezogen auf die jeweilige räumliche Ausdehnung, gesteigert und eine bessere Isolation erreicht. Eine Herabsetzung der Nesttemperatur wird durch die Verminderung der Arbeiterinnendichte erzielt. Diese Regulationsleistung beruht fast ausschliesslich auf der Ausnutzung der durch den Stoffwechsel bedingten "Abfallwärme ».

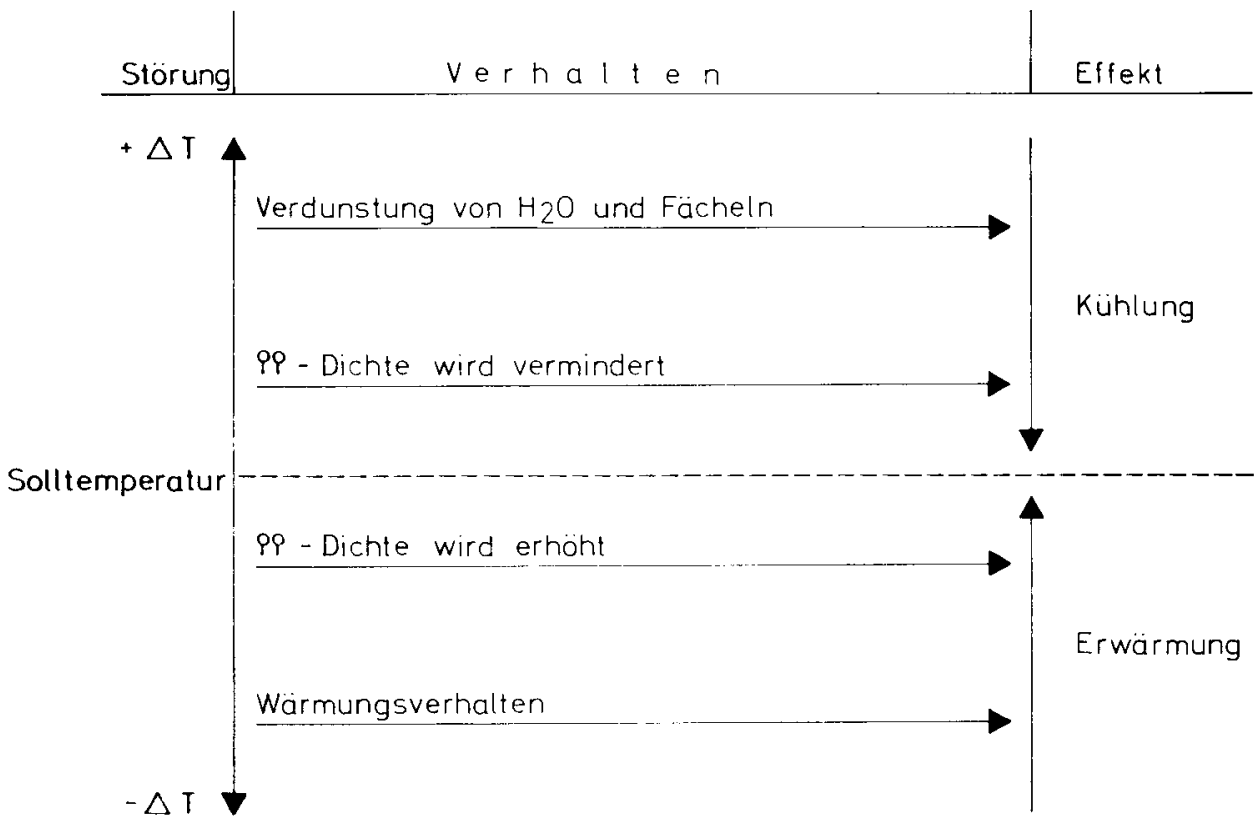

Авв. 6. - Temperaturregulationsschema.

FIG. 6. - Schéma de la régulation thermique.

Bei massiveren Störungen kann die Solltemperatur nur mit Massnahmen aufrecht erhalten werden, die zusätzliche, speziell auf die Temperaturregulierung gerichtete 
Arbeit erfordern. Bei beträchtlichen Erhitzungen, etwa der Sonneneinstrahlung, wird von den Arbeiterinnen Wasser eingetragen Lindauer (1954 b). Die Verdunstung dieses Wassers im Nest wird durch Fächeln beschleunigt, eine spezielle Flügelbewegung zur Steigerung der Luftzirkulation. Die entstehende Verdunstungskälte bewirkt dann eine Kühlung.

Bei stärkerem Abweichen von der Solltemperatur nach unten, etwa in kalten Nächten während des Spätsommers, tritt Wärmungsverhalten auf. Die Arbeiterinnen sind in der Lage, mit Hilfe ihrer umfangreichen Flugmuskulatur beträchtliche Wärmemengen zu erzeugen, und so einer Unterkühlung der speziell in dieser Hinsicht empfindlichen Puppen entgegenzuwirken. Durch die spezifischen Signale der Puppe, gerichtet auf zwei verschiedene Sinne, wird erreicht, dass die Wärmeproduktion in rationeller Weise auf den Bereich des aktuellen Bedarfs beschränkt bleibt.

Eingegangen im Juni 1978

Reçu pour publication en juin 1978

\section{RÉSUMÉ}

\section{Introduction}

Il existe de nombreuses données, en partie exacte (Gates 1914, Hess 1926, Himmer 1927 a, Free + SPENCER-BOoth 1958, BüDEL + Herold 1960) sur la répartition des températures à l'intérieur de la colonie selon les conditions extérieures. Les bases éthophysiologiques de la thermorégulation sont pourtant peu connues à ce jour. Ainsi on ne dipose d'aucune donnée sur le comportement de chauffage des abeilles dans la colonie, bien qu'il existe des observations très précises sur les travaux habituels des jeunes abeilles à l'intérieur de la ruche. Les causes ne sont pas claires, sur lesquelles repose la différence dans la répartition des températures entre une colonie hivernale et une colonie estivale. Une hypothèse, basée sur des mesures comparatives de colonie avec couvain et sans couvain (RITTER 1975), a été émise selon laquelle la répartition des températures dans la colonie durant l'été est déterminée par un chauffage actif du couvain operculé par les ouvrières, dans la mesure où la température basse extérieure représente un danger de refroidissement. On doit par conséquent tenter d'utiliser le comportement de chauffage des ouvrières comme indicateur pour reconnaître le couvain.

\section{Matériels et méthodes}

Les tests ont été conçus comme des expériences de choix simultané. On a découpé dans un rayon à miel les deux échantillons de couvain à tester et placé ensuite ce rayon dans une cage grillagée. Sur les 2 échantillons, ainsi que sur le rayon entre les échantillons, on a placé des thermocouples dont la température est enregistrée durant l'expérience par un stylet (Fa. Hartmann + Braun, Polycomb II). Dans les cages ont été introduites de jeunes ouvrières (Apis mellifera carnica), qui avaient été prélevées sur 2 à 4 rayons d'une colonie. Sur la figure 1 est représenté le déroulement type d'une expérience, sur la figure 2 la répartition des températures enregistrées.

\section{Résultats}

Attractivité

Les cellules de couvain operculé ont été constamment chauffées de préférence au couvain non operculé. En présence de cellules operculées d'ouvrières et de cellules operculées de mâles, les ouvrières ont chauffé ces dernières. Ce n'est que pour une proportion de 5 cellules d'ouvrières pour 1 cellule de mâle que les cellules d'ouvrières ont été chauffées dans 4 cas sur 7. Dans une expérience renouvelée 14 fois on a offert aux abeilles 350 cellules operculées d'ouvrières et 2 cellules royales operculées et intactes. Dans tous les cas les cellules royales ont été chauffées. 
Analyse de la "reconnaissance" des cellules royales

Si l'on présente des cellules royales vides avec 350 cellules d'ouvrières, ce sont ces dernières qui sont chauffées (fig. 1) mais si, un peu avant le début de l'expérience, on substitue à la pupe royale vivante une pierre de même poids, les abeilles chauffent cette "cellule de pierre ». Des blocs légers de polystyrène expansé ont été placés dans les cellules royales. Selon la position du corps en polystyrène, les cellules ont été (fig. 4, à gauche), ou n'ont pas été (fig. 4, à droite) chauffées.

L'hypothèse, selon laquelle la tension mécanique de la paroi de la cellule joue un rôle dans la reconnaissance de la cellule royale, a pu être confirmée par les expériences avec les "pupes-pendules » (fig. 5). Ces cellules n'ont pas été chauffées.

Le moment du retrait de la pulpe avant le début de l'expérience exerce également une action décisive sur la stimulation. Des cellules royales, maintenues "vides " plus de 4 heures à l'étuve à $34^{\circ} \mathrm{C}$, n'ont déclenché, une fois munies de la pierre adéquate, aucun comportement de chauffage. Des pupes vivantes, conservées dans les cellules pendant 2 jours à $34^{\circ} \mathrm{C}$, ont permis de les réactiver. On a prouvé l'existence d'un facteur chimique lors de la reconnaissance des cellules royales en réactivant, à l'aide d'extraits de pupes, des cellules auparavant inactives.

\section{LITERATURNACHWEIS}

ARistoteles, 1919. - Tierkunde V, Buch-Kap. 22-554 b aus : Klek und Armbruster " Die Bienenkunde des Altertums " I. Archiv Bienenkd., 1 (6), 185-240.

Büdel A., Herold E., 1960. - Biene und Bienenzucht., München, Ehrenwirth Verlag.

EscH H., 1968. - Über die Körpertemperaturen und den Wärmehaushalt von Apis mellifica Z. vergleich. Physiol., 43, 305-335.

Free J.B., Spencer-Booth Y., 1958. - Observations on the temperature regulation and food consumption of honeybees (Apis mellifera). J. exp. Biol., 35 (4), 930-937.

Gates B. N., 1914. - The temperature of the bee colony. Bull. U.S. Dept. Agric., 96, 1-29.

Hess W. R., 1926. - Die Temperaturregulierung im Bienenvolk. Z. vergleich. Physiol., 4, 465-487.

Himmer A., 1927 a. - Der soziale Wärmehaushalt der Honigbiene. Il. Die Wärme der Bienenbrut. Erlanger Jb. Bienenkd., 5, 1-32.

IsHAY J., 1972. - Thermoregulatory pheromones in wasps. Experientia, 28, 1185-1187.

Lindauer M., 1952. - Ein Beitrag zur Frage der Arbeitsteilung im Bienenstaat. Z. vergleich. Physiol., 34 (4), 299-345.

Lindauer M., 1954 b. - Temperaturregulierung und Wasserhaushalt im Bienenstaat. Z. vergleich. Physiol., 36 (4), 391-432.

Martin H. und Lindauer M., 1966. - Sinnesphysiologische Leistungen beim Wabenbau der Honigbiene. Z. vergleich. Physiol., 53, 372-404.

RITTER W., 1975. - Wärmeerzeugung von Bienenvölkern (Apis mellifica L.). Diplomarbeit Inst. für Bienenkunde, Fachbereich Biologie der J. W. Gæthe Univ., Frankfurt a. M.

RitTer W. und KoEniger N., 1977. - Influence of the brood on the thermoregulation of honeybee colonies. Proc. VIII Congr. IUSSI Wageningen, 283-284.

RÖsch G. A., 1925. - Untersuchungen über die Arbeitsteilung im Bienenstaat 1. Teil. Die Tätigkeiten im normalen Bienenstaate und ihre Beziehungen zum Alter der Arbeitsbienen. $Z$. vergleich. Physiol., 2 (6), 571-631.

WeIss K., 1962. - Über die Lebensfähigkeit von offener und gedeckelter Brut ausserhalb des Bienenvolkes. Z. Bienenforsch., 6 (4), 104-114. 